\title{
HOSPITAIS E PROGRAMAS DE QUALIDADE NO ESTADO DE SÃO PAULO
}

\author{
Ana Maria Malik \\ Médica, Mestre em Administração pela FGV-EAESP, Doutora em Medicina pela FMUSP, Professora do Departamento \\ de Administração Geral e Recursos Humanos da FGV-EAESP e Diretora do Proahsa-FGV. \\ E-mail: anamalik@fgvsp.br \\ João Pedro Teles \\ Dentista, Mestrando em Administração de Empresas, concentração \\ em Administração Hospitalar e de Sistemas de Saúde, na FGV-EAESP. \\ E-mail: jpteles@gvmail.br
}

\begin{abstract}
RESUMO
A área da Administração em Saúde tem uma visão e uma prática relativas à qualidade diferentes daquelas observadas na indústria. Este artigo apresenta levantamento realizado em amostra de 159 hospitais do estado de São Paulo, no segundo semestre de 1999, quanto à implantação ou não de iniciativas de qualidade. Foram estudados hospitais públicos, filantrópicos, não filantrópicos e universitários. Dos 97 hospitais que responderam à pesquisa, 23\% afirmavam ter alguma iniciativa desse teor. Os 77\%, cuja resposta foi negativa, atribuíam essa decisão aos custos dos programas, à demora na obtenção de resultados e à falta de necessidade. Muitos dos que apresentaram iniciativas indicaram pouco controle sobre os custos incorridos e outros tipos de conseqüências. Quase todos os que responderam positivamente informaram monitorar seus indicadores gerenciais.
\end{abstract}

\section{ABSTRACT}

The Health Administration field understands and deals with quality issues in a way that differs from what is seen in the industry. This article presents a survey done in a sample of 159 hospitals in São Paulo state during the second semester 1999 regarding the implementation of quality initiatives. The sample had public, philanthropic, non-philanthropic and university hospitals. Among 97 hospitals answering the survey, 23\% said they had some quality initiatives. The other 77\%, whose answer was negative, blamed high costs, delayed results and lack of need for that fact. Most of those that had the initiatives did not know much about their costs and their consequences. Almost all of those that answered positively said they monitored managerial indicators.

\section{PALAVRAS-CHAVE}

Qualidade, gestão de qualidade em hospitais, gestão hospitalar, mudança organizacional, gestão de saúde.

Quality, quality management in hospitals, hospital management, organizational change, health management. 


\section{INTRODUÇÃO}

A discussão contemporânea na área de Administração em Saúde inclui aspectos referentes a competitividade, alianças estratégicas, parcerias e outras questões, em geral introduzidas pela leitura de textos e pelo conhecimento da realidade norte-americana (Ferreira, 2000). Nos EUA, desde os anos 80, o modelo de mercado assumido pelo setor vem questionando paradigmas ou valores aceitos universalmente nas sociedades latinas. Entre eles está

\section{A ASSISTÊNCIA DOMICILIAR, A} TELEMEDICINA, AS NOVAS DROGAS

\section{E MAIS ACESSO A CONHECIMENTO}

\section{TORNARAM OBSOLETOS ALGUNS}

\section{INDICADORES HOSPITALARES}

\section{UTILIZADOS DESDE O FINAL}

\section{dA II Guerra Mundial}

\section{nA AmÉrica no Norte.}

o de acesso, exemplificado no discurso dos profissionais de saúde com frases como "cada caso é um caso" ou "saúde não tem preço". Relativizações do tipo "saúde não tem preço, mas tem custo" e perguntas do tipo "como compatibilizar equiidade com eficiência" levantam novas questões, principalmente visando entender de onde viriam a sobrevivência dos hospitais e demais serviços de saúde, sua viabilidade econômico-financeira e, conseqüentemente, de onde sairia seu financiamento, especialmente num modelo de mercado, visto por muitos como uma forte tendência para a realidade brasileira.

No setor social, ainda não está respondida a pergunta relativa a quais os resultados a serem perseguidos, "eqüidade, eficiência ou saúde"? A substituição da categoria cure pela categoria care é aceitável em função de que valores? O usuário do sistema de saúde tem a expectativa de ser atendido por profissionais qualificados, que indiquem condutas baseadas em conhecimento sólido. Por isso, receber tratamentos sem efeitos colaterais não significa para eles qualidade no tratamento. Resultados clínicos (como mudanças objetivas no estado físico ou nos resultados de exames complementares) não são equivalentes a ganhos em bem-estar nem a benefício social pela sobrevida ou até pela cura.

Muitos dos efeitos do progresso das ciências médicas (ciências da vida), como o aumento puro e simples da sobrevida ou como menores taxas de mortalidade por agravos antigamente fatais, geram custos em função da manutenção de vidas mais longas ou do surgimento de novas necessidades. Há quem discuta essas novas disponibilidades em termos de satisfação ou custo/benefício e há quem se refira a esses ganhos como qualidade.

Para garantir a sobrevivência dos hospitais, é necessário garantir clientela. Os clientes podem ser os usuários diretos, os médicos ou os agentes financiadores. No Brasil, em 2001, tratar de financiamento em saúde significa, em, no mínimo, 70\% dos casos, mencionar SUS. Em termos de população coberta, os atores da chamada Saúde Suplementar ainda não mostram evidências de atingir mais que $30 \%$ a $35 \%$ da população. No Brasil, a população, em pelo menos $70 \%$ dos casos, dirige-se aos serviços financiados pelo SUS, ou seja, a serviços que oferecem cobertura universal. Uma estratégia para aumentar o número de clientes, desde que isso se torne necessário, pode ser mostrar evidências de que o serviço oferecido apresenta qualidade, independente do que isso signifique.

Dados observados nos hospitais privados financiados por fontes privadas, que atendem pacientes com cobertura de Saúde Suplementar ou aqueles, cada vez mais raros, que pagam pelos procedimentos utilizados de seu próprio bolso, contestam a decantada realidade norte-americana, de baixos indicadores de utilização de leitos hospitalares. No município de São Paulo, durante o ano 2000, quase todos os hospitais considerados de alto custo, alto preço e boa qualidade apresentaram taxa de ocupação próxima de $90 \%$. Em alguns hospitais financiados pelo SUS e em algumas especialidades, pode-se falar em aumento de eficiência. Por exemplo, dados do DATASUS mostram redução na taxa de ocupação, associada a melhoras nas médias de permanência. Ao mesmo tempo, devido a algumas distorções do modelo de financiamento, ainda há demanda reprimida em especialidades críticas, como Terapia Intensiva (de Adultos, Pediátrica e Neonatal) e Oncologia, entre outras.

Existe uma tendência, internacionalmente observada, de redução na necessidade por leitos hospitalares. Isso obriga a analisar os indicadores nacionais disponíveis atualmente e a considerar tendências internacionais do setor e a introdução de inovações tecnológicas, cada vez mais rápidas. Se os parâmetros atuais de planejamento continuarem a ser utilizados, corre-se o risco de realizar investimentos, públicos ou privados, de necessidade discutível, por pressão de corporações ou outros grupos de interesse.

A assistência domiciliar, a telemedicina, as novas drogas e mais acesso a conhecimento tornaram obsoletos alguns indicadores hospitalares utilizados desde o final da II Guerra Mundial na América no Norte (EUA e Canadá). No Brasil, embora de maneira mais lenta, também se podem observar mudanças nos valores dos indicadores preconizados (inclusive na proposta apresentada para con- 
sulta pública pelo Ministério da Saúde, no final de 2000, no seu site). O primeiro passo para perceber a mudança é olhar para os indicadores. Isso vale para os procedimentos de administração, os de informação médica ou de epidemiologia hospitalar, que permitem acompanhar - pelo menos em cada hospital ou nos serviços financiados pelo mesmo caixa - a evolução no tipo de paciente (gravidade), tipo de doença (diagnóstico) e tipo de cuidado (evolução). Esses dados fazem parte da caracterização do chamado case mix, já incorporado como variável em estudos acadêmicos no Brasil e em planejamentos nos EUA, mas certamente ainda não consagrado em nossos serviços.

Nesse cenário, começou-se no país, timidamente desde os anos 70, com um pouco mais de frequiência nos anos 80 e como um certo modismo nos anos 90 , a se falar em qualidade na área de saúde/hospitalar. Nos anos 90, a quantidade de cursos, publicações e organizações não governamentais que passaram a se interessar pelo assunto tomou vulto, o que se torna evidente em virtude da quantidade de material impresso, seminários, trabalhos acadêmicos e modelos de consultoria disponíveis (Schiesari, 1999; Malik e Teles, 2000).

No estado de São Paulo, há um grande número de hospitais, públicos e privados, e grande disponibilidade de cursos, editoras e seminários na área. Observa-se concentração de todo tipo de recursos humanos, financeiros, materiais, tecnológicos, e encontram-se mais universidades e serviços de saúde. Existe ainda o mito de que (embora não se comente quanto aos piores) está em São Paulo a maior parte dos melhores serviços de saúde do Brasil. A imprensa - também nesse estado - não pára de falar da crise da saúde, da falta de qualidade do setor e da sua ineficiência. Ao mesmo tempo, é para onde se deslocam muitos brasileiros em busca de assistência para seus males, de emprego para suas habilitações e de educação continuada para sua atividade profissional.

A questão que levou à realização deste artigo foi a de que haveria, no estado de São Paulo, hospitais com programas/atividades voltados para a qualidade. Isso posto, seria desejável descobrir quais os programas ou atividades mais freqüentemente utilizados e como jogar luz sobre alguns dos mitos que envolvem o assunto.

\section{ESPECIFICIDADES DA ÁREA DE SAÚDE}

Sem dúvida, podem-se mencionar algumas especificidades existentes na área da saúde quando se discute qualidade (Malik, 1992). Há argumentos sobre as chamadas características das organizações de saúde em livros clássicos de Comportamento Organizacional em Saúde e citados em artigos nacionais (Shortell e
Kaluzny, 1988; Azevedo, 1995). Ocorrem há anos discussões na área de avaliação e de administração da qualidade quanto a se esta é utilizável apenas no setor industrial ou também se aplica à área da saúde (Malik e Schiesari, 1998).

Características do setor ajudam a confundir variáveis envolvidas na avaliação dos resultados e, por conseguinte, dos efeitos que programas de melhoria podem trazer. Por exemplo, poucos profissionais com formação administrativa ousam discutir com médicos sobre a afirmação de que os procedimentos diagnósticos ou terapêuticos são prescritos com vistas ao bem-estar do paciente (independente do tipo de financiamento do serviço) ou de que determinada despesa deve ser feita, sob pena de provocar a morte de algum paciente, por culpa do administrador.

\section{Pesquisas acadêmicas}

\section{MOSTRAM QUE POUCOS}

\section{SERVIÇOS DE SAÚDE DISPÕEM DE INDICADORES E MENOS}

\section{AINDA OS UTILIZAM.}

Pesquisas acadêmicas mostram que poucos serviços de saúde dispõem de indicadores e menos ainda os utilizam (Bittar, 1997; Silva, 1998). Existem publicações que se propõem a divulgar aos gestores hospitalares alguns indicadores com intenção de permitir comparação entre diferentes serviços (a Unisinos sedia um grupo de hospitais na Região Metropolitana de Porto Alegre que faz análise de seus indicadores e os Indicadores Proahsa divulgam dados retirados do DATASUS, de uma amostra para dados financeiros, de outra amostra para dados sobre RH e de uma amostra de hospitais inscritos em um programa de certificação para dados sobre "qualidade"). Em todos os casos, pode haver dúvidas quanto à metodologia de coleta, tipo de amostra e validade dos dados para uma utilização mais ampla. A prática de trabalhar com indicadores é tão pouco usual, que muitos hospitais consideram essas oportunidades de olhar para dados de outros como benchmarking, independente da existência de qualquer semelhança entre os serviços, da finalidade da divulgação dos dados e de sua real utilidade. Essa oportunidade também não os incentiva a mudar de comportamento.

Há características das organizações de saúde que tornam difícil internamente a avaliação de resultados nelas obtidos. Parte-se da premissa de que seus trabalhadores fazem o melhor que pode ser feito. Esse argu- 
mento tem relação com algum grau de autoritarismo e de corporativismo (Carapinheiro,1993), pois assume a assimetria na relação equipe/paciente (ou médico/paciente).

Isso auxilia a explicar o fato de haver hospitais em que se consegue modificar a cultura de todas as áreas (por exemplo, em relação à informática) com maior ou menor facilidade (entre as mais difíceis está a área médica/ assistencial) e outros em que ocorre refluxo das iniciativas (Picchiai, 1998). Hoje se aceita que é possível transformar instrumentos de racionalização em trunfos voltados para a melhoria de resultados de atendimento (Azevedo e Korycan, 1999).

O corporativismo é uma das causas da existência, em hospitais, de pelo menos duas instâncias de comando simultâneo, a funcional e a por projeto (Gonçalves, 1998). A liderança do grupo profissional nuclear pode dificultar ações coordenadas, caso opte por seguir/definir rumos próprios. Observa-se que pelo menos duas áreas dos hospitais (a enfermagem e o corpo médico) usualmente desenham seus próprios modelos de gestão e de avaliação, não aceitando os ditames mais gerais da organização. Esse tipo de configuração questiona o critério de "totalidade" previsto por Deming, em sua gestão da qualidade total (Deming, 1990).

Atualmente, ocorre uma discussão em relação aos diferentes modelos universalmente aceitos de qualidade (Malcolm Baldrige, Deming Prize, European Quality Award): talvez não deva existir uma única receita para as organizações interessadas em melhorar seu desempenho (Harrington, 1997). As conclusões deste artigo podem ser extrapoladas para o brasileiro Prêmio Nacional da Qualidade, que tardiamente vem chegando ao setor saúde como forma de torná-lo comparável às demais áreas de atividade econômica. Ele é por vezes defendido como modelo de gestão, assumindo como premissa que a área de saúde/hospitalar não dispõe de seus próprios instrumentos. A busca pelo aprimoramento, na prestação de serviços ou na gestão, deve ser estimulada. A forma de chegar a ela pode ser diferente.

\section{METODOLOGIA}

De novembro de 1998 a janeiro de 1999, foi realizado um estudo piloto com executivos de hospitais e outros serviços de saúde nos municípios de São Paulo e do Rio de Janeiro. Esse estudo, realizado com hospitais cujos executivos mantinham algum tipo de relação com os pesquisadores, tinha como objetivo identificar respostas comuns e pontos considerados importantes pelos respondentes que servissem de substrato para a elaboração do questionário utilizado para a investigação atual. Para tanto, foram enviados, por fax, 13 questionários com questões abertas relacionadas a programas e atividades de qualidade nos serviços selecionados.

Apenas 10 dos 13 questionários retornaram preenchidos $(80 \%)$, o que fez crer que haveria dificuldade em obter mais respostas numa situação de amostra probabilística, com hospitais e dirigentes não conhecidos. Foi elaborado, então, o questionário utilizado neste trabalho. Optouse por enviar um questionário com questões fechadas pelo correio, a fim de poder trabalhar com uma amostra de todo o estado, e não apenas de uma região.

Em paralelo com a elaboração da ferramenta de pesquisa, deu-se início ao processo de determinação e seleção da amostra. Com base no banco de dados do Centro de Informações de Saúde da Secretaria de Estado da Saúde de São Paulo, obteve-se o Cadastro Geral dos Hospitais de São Paulo atualizado em 1998 (CIS, 1998). Nesse cadastro, os 956 hospitais do estado são apresentados subdivididos em categorias segundo propriedade/regime jurídico (federais, estaduais, municipais, universitários com e sem pesquisa, filantrópicos, privados com finalidade lucrativa, conveniados, contratados e próprios). Buscando maior homogeneização, tais categorias foram reagrupadas em públicos (subdivididos entre federais, estaduais e municipais), filantrópicos, não filantrópicos (agrupados os hospitais privados com finalidade lucrativa, conveniados, contratados e próprios) e universitários (com ou sem pesquisa).

Um segundo critério utilizado para a classificação dos hospitais foi o porte. Embora o Ministério da Saúde pre-

Tabela 1 - Distribuição dos hospitais do estado de São Paulo por tipo e porte

\begin{tabular}{|l|c|c|c|c|c|}
\hline \multirow{2}{*}{ Descrição } & \multicolumn{5}{|c|}{ Universo } \\
\cline { 2 - 7 } & Até $\mathbf{5 0}$ & $\mathbf{5 1}$ a $\mathbf{1 5 0}$ & Mais de $\mathbf{1 5 0}$ & TOTAL & $\%$ do universo \\
\hline Público & 110 & 35 & 47 & $\mathbf{1 9 2}$ & $\mathbf{2 0 , 1} \%$ \\
\hline Filantrópico & 88 & 166 & 101 & $\mathbf{3 5 5}$ & $\mathbf{3 7}, \mathbf{1 \%}$ \\
\hline Não filantrópico & 134 & 138 & 111 & $\mathbf{3 8 3}$ & $\mathbf{4 0 , 1} \%$ \\
\hline Universitário & 0 & 7 & 19 & $\mathbf{2 6}$ & $\mathbf{2 , 7} \%$ \\
\hline Total & $\mathbf{3 3 2}$ & $\mathbf{3 4 6}$ & $\mathbf{2 7 8}$ & $\mathbf{9 5 6}$ & $\mathbf{1 0 0 , 0} \%$ \\
\hline Porcentagem & $34,7 \%$ & $36,2 \%$ & $29,1 \%$ & $\mathbf{1 0 0} \%$ & - \\
\hline
\end{tabular}

Fonte: CIS, Secretaria de Estado da Saúde/São Paulo, 1998. 
conize a divisão pelas categorias "pequeno" $(0$ a 50 leitos), "médio" (51 a 150 leitos), "grande" (151 a 300 leitos) e "especial" (acima de 300 leitos), para esta pesquisa, uma vez que o total de hospitais nos dois primeiros grupos é semelhante e o agrupamento das duas últimas categorias se aproxima das duas anteriores, optou-se por trabalhar com apenas três. Dessa forma, o universo dos hospitais do estado de São Paulo ficou distribuído conforme a Tabela 1.

Utilizando como base para os cálculos a metodologia de amostragem aleatória simples e assumindo um erro amostral de 0,10 com um nível de confiança de 95\%, seria necessária uma amostra de cerca de 100 hospitais. Supondo um índice de respostas próximo de $80 \%$, determinou-se o tamanho da amostra em 120 unidades.

No processo de amostragem, os estratos selecionados foram hospitais públicos, filantrópicos, não filantrópicos e universitários, agrupados de acordo com o critério anteriormente descrito. Dentro de cada estrato, os hospitais foram distribuídos de forma aleatória em uma planilha Excel, para serem selecionados. A distribuição da amostra planejada pode ser verificada na Tabela 2.

Foram feitos três envios do questionário, a fim de aumentar o número de respondentes. O primeiro foi feito no início de setembro de 1999, o segundo, no final de outubro e o terceiro, no início de dezembro. A resposta espontânea ao primeiro envio foi de $12 \%$ (19 questionários).

Após as cobranças, esse índice subiu para 23\% (36 questionários). Para o segundo envio, foi obtido um índice de resposta similar, atingindo o número de 66 questionários $(42 \%)$. Como qualquer nova resposta obtida corresponderia a quase $1 \%$, decidiu-se tentar chegar a pelo menos $50 \%$. Assim, partiu-se para o terceiro envio, por meio do qual foi alcançado o número de 97 respondentes, atingindo $61 \%$ da amostra.

\section{RESULTADOS}

\section{Caracterização dos resultados}

Os hospitais respondentes não corresponderam exatamente à amostra planejada. Por exemplo, os hospitais de pequeno porte, dos quais se esperava $33,3 \%$ dos respondentes, representaram apenas $27,8 \%$ das respostas. Os de médio porte, que deveriam ser $36,5 \%$, foram $41,2 \%$, os de grande porte e de porte especial, que deveriam ser $30,2 \%$, foram $30,9 \%$, ou seja, os hospitais de pequeno porte terminaram por ser sub-representados, os de médio porte tiveram proporcionalmente maior representação e os de grande porte/porte especial responderam em porcentagem mais próxima daquela esperada.

Os hospitais públicos, que representam $20,1 \%$ do universo (e 19,5\% da amostra), entre os respondentes, foram $27,8 \%$, mostrando talvez maior disponibilidade em oferecer seus dados para estudo. $\mathrm{O}$ mesmo pode ser observado nos hospitais filantrópicos e nos universitários. Essa grande disponibilidade foi contrabalançada pelos hospitais ditos "não filantrópicos", que representam $40,1 \%$ do universo (40,3\% da amostra), mas que, entre os respondentes, passaram para $26,8 \%$.

Dos 97 hospitais respondentes, $70(72,2 \%)$ afirmaram conhecer algum sistema de qualidade. Isso significa que $27,8 \%$ dos respondentes, dirigentes hospitalares, informaram não conhecer nenhum no estado de São Paulo em 1999. Esse dado é preocupante, pois o fato de mais de um quarto dos respondentes não conhecer sistemas de qualidade significa não se interessar pela imprensa diária, que menciona programas e sistemas de qualidade com intenção de divulgação (e, nos anos 90, essa área de conhecimento foi muito divulgada). Não conhecer sistemas de qualidade também representa não olhar para as ofertas de cursos e seminários, do setor e fora dele.

O total de citações sobre sistemas de qualidade conhecidos foi 199. Destas, 132 referiam-se a sistemas não específicos para o setor saúde e 63 a sistemas específicos do setor. As quatro citações restantes foram "outros". Essa resposta aponta para a baixa credibilidade dos instrumentos disponíveis para a saúde mesmo dentro do setor e, certamente, para a maior divulgação dos instrumentos/ métodos de gestão industriais.

Nessa mesma população de 97 respondentes, perante a pergunta sobre se conheciam algum caso de implantação bem-sucedida de programa de qualidade, oito não

Tabela 2 - Distribuição dos hospitais da amostra planejada por tipo e porte

\begin{tabular}{|l|c|c|c|c|c|}
\hline \multirow{2}{*}{ Descrição } & \multicolumn{5}{|c|}{ Númostra planejada } \\
\cline { 2 - 7 } & Até $\mathbf{5 0}$ & $\mathbf{5 1}$ a $\mathbf{1 5 0}$ & Mais de $\mathbf{1 5 0}$ & \multicolumn{1}{c|}{ TOTAL } & $\%$ da amostra \\
\hline Público & 17 & 6 & 8 & $\mathbf{3 1}$ & $\mathbf{1 9 , 5 \%}$ \\
\hline Filantrópico & 14 & 28 & 17 & $\mathbf{5 9}$ & $\mathbf{3 7}, \mathbf{1 \%}$ \\
\hline Não filantrópico & 22 & 23 & 19 & $\mathbf{6 4}$ & $\mathbf{4 0 , 3} \%$ \\
\hline Universitário & 0 & 1 & 4 & $\mathbf{5}$ & $\mathbf{3}, \mathbf{1 \%}$ \\
\hline Total & $\mathbf{5 3}$ & $\mathbf{5 8}$ & $\mathbf{4 8}$ & $\mathbf{1 0 0} \%$ & $\mathbf{1 0 0 , 0} \%$ \\
\hline Porcentagem & $33,3 \%$ & $36,5 \%$ & $30,2 \%$ & - \\
\hline
\end{tabular}


responderam $(8,2 \%)$. Dos demais, 44 responderam que sim, o que significa que mais de $50 \%$ dos que responderam a essa pergunta afirmaram não conhecer nenhum caso de programa de qualidade bem-sucedido. Dos 44 que responderam que sim, houve dispersão das respostas, tanto

\section{A PRÁTICA DE TRABALHAR COM INDICADORES É TÃO POUCO USUAL,} QUE MUITOS HOSPITAIS CONSIDERAM ESSAS OPORTUNIDADES DE OLHAR PARA DADOS DE OUTROS COMO BENCHMARKING, INDEPENDENTE DA EXISTÊNCIA DE QUALQUER SEMELHANÇA ENTRE OS SERVIÇOS, DA FINALIDADE DA DIVULGAÇÃO

\section{DOS DADOS E DE SUA REAL UTILIDADE.}

na área de saúde quanto na área empresarial. Esse pode ser um exemplo da distância entre o que se lê/estuda e o que de fato se implanta, freqüentemente observada na gestão de saúde (e nos cursos de administração para gerentes de saúde).

Quanto à pergunta sobre o conhecimento de alguma tentativa de implantação que tenha sido abandonada, nos 97 questionários recebidos, não havia resposta em 11 $(11,3 \%)$. Dos 86 que responderam, 11 afirmaram conhecer fracassos (seis na área da saúde - tanto em hospitais públicos quanto em hospitais privados - e cinco no setor industrial). Pouco mais de $10 \%$ de fracassos reconhecidos não é marca alarmante e, certamente, esse não deve ser motivo para o baixo número de adesão dos respondentes a iniciativas de qualidade.

Retomando a questão que motivou esta pesquisa, em relação à existência ou não de iniciativas de qualidade em hospitais do estado de São Paulo, dos 97 respondentes, 22 $(22,7 \%)$ afirmaram que no hospital onde trabalham já foi implantado ou está em implantação algum(a) sistema/iniciativa de qualidade (e, consequientemente, 75 - ou $77,3 \%$ - informaram que não).

\section{Hospitais com implantação}

de sistema/iniciativa de qualidade

Vinte e dois hospitais $(22,7 \%)$ apresentaram-se como tendo implantado algum sistema ou iniciativa de qualidade. A distribuição desses 22 hospitais era: sete públi$\cos$, cinco filantrópicos, oito não filantrópicos e dois uni- versitários. Entre eles, 1 era de pequeno porte, 8, de médio porte e 13 faziam parte da categoria "acima de 150 leitos" usada neste estudo.

Os 22 respondentes de hospitais que citaram implantação de programas de qualidade mencionaram 29 sistemas ou iniciativas. Dos 29 sistemas mencionados, 15 foram específicos do setor. Dessa forma, quando o conhecimento se converte em ação, parece que se passa a valorizar mais as especificidades do setor (em mais de 50\% dos casos, implantaram-se programas desenvolvidos para o setor). A resposta para o tempo de implantação mostrou uma distribuição em que 3 disseram ter levado menos de um ano para a implantação, 5 deles de um a dois anos, 3 entre dois e três anos e os outros 11 declararam estar ainda em processo de implantação. Nenhum deles relatou tempo de implantação maior que três anos. A integralidade do processo, o grau de completude e a avaliação da manutenção não foram considerados. As razões mais valorizadas para a implantação foram, em primeiro lugar, melhorar a assistência prestada, em segundo, melhorar a organização e, em terceiro, aumentar a satisfação do usuário.

Embora apenas em terceiro lugar, o usuário foi valorizado. A explicação para isso é que não só questões voltadas à satisfação da burocracia levaram a essa situação ou pode-se pensar que o discurso "politicamente correto" obriga a mencionar o paciente.

Em 12 respostas (de 22), a direção superior foi percebida como responsável pela seleção do sistema implantado. Os motivos para a seleção foram, em primeiro lugar, informações obtidas em cursos ou eventos científicos, em segundo, informações da literatura e, em terceiro, determinação superior.

Analisando as respostas obtidas quanto aos custos de implantação, tanto nos gastos com treinamento quanto naqueles com informatização e com ambiente físico, a mais freqüente foi "não sei". Nos gastos com consultoria, a alternativa "não sei" foi a segunda mais citada (a primeira foi "zero"). Em geral, os respondentes tenderam a minimizar os custos: valores abaixo de $\mathrm{R} \$ 10.000,00$ sempre estavam entre as alternativas mais citadas enquanto valores acima de $R \$ 100.000,00$ eram mencionados com frequiência menor. No setor saúde, cujos custos são altos e receitas são sempre consideradas insuficientes, o desconhecimento profundo a respeito de custos de diferentes programas é preocupante para os administradores e para os financiadores.

Quanto à pergunta relativa às facilidades encontradas na implantação, entre as respostas obtidas, a mais valorizada foi o envolvimento da direção superior, seguida pelo interesse do corpo funcional pela qualidade e pela atitude favorável às mudanças por parte do corpo funcional.

Numa área na qual o discurso tradicional é a resistência à mudança, essa "atitude favorável" parece surpreendente ou mostra-se a favor da tentativa de mudar uma situação da 
qual as pessoas estão cansadas. Cada um está convencido de que sabe fazer sua parte, mas se frustra pela quantidade de restrições que atribui aos outros ou à organização.

Em relação às dificuldades, a maior foi a falta de formação do pessoal envolvido, seguida, pela ordem, por tempo decorrido até a obtenção dos resultados, peculiaridades do setor saúde, que conflitariam com sistemas de qualidade, custos elevados, resistência dos funcionários, dificuldade de envolvimento do corpo funcional, dificuldade de envolvimento da direção superior e resistência dos gerentes.

Vinte, dentre os vinte e dois hospitais que declaram ter programas de qualidade, afirmaram monitorar indicadores, em confronto flagrante com os achados de Silva (1998), para quem hospitais não estão interessados no assunto. Como aqui se trata da minoria que tem programas, eles já constituem um grupo diferenciado. Pode estar aí a explicação para a discrepância. Das 58 respostas quanto aos tipos de indicadores, $29,3 \%$ referem-se a indicadores de produtividade, $24,1 \%$ mencionam indicadores financeiros, $22,4 \%$ indicam os de recursos humanos, $20,7 \%$ citam indicadores de qualidade e $3,4 \%$ referem-se a indicadores de outro tipo.

Os resultados positivos obtidos foram, pela ordem, a participação dos funcionários, o aumento da satisfação do usuário (essa resposta reforça uma das causas alegadas para a implantação), empatados com a racionalização dos processos e a responsabilização dos funcionários e a melhora nos indicadores de eficiência. A não-percepção de resultados até o momento da pesquisa teve mais respostas do que a redução de custos. Quanto aos resultados negativos, o mais citado foi a ausência de resultados até o momento da pesquisa, seguido pelo aumento de conflitos internos.

Esses são dois motivos de justificada preocupação, pois, havendo ou não conhecimento sobre custos, devemse justificar gastos com resultados e de alguma espécie e poucos são os administradores que conseguem considerar os conflitos como fator de crescimento da organização. Em terceiro lugar, apareceu a concentração no sistema de qualidade em detrimento das pessoas; a seguir, o aumento da competição interna. $\mathrm{O}$ aumento de custos foi o próximo e ainda apareceu a redução da satisfação dos funcionários e dos usuários. Dezenove responderam sobre resultados inesperados: nove disseram tê-los observado e dez não.

\section{Hospitais sem implantação de sistema/iniciativa de qualidade}

Dos 75 hospitais nessa categoria, em 1 tinha sido tomada a decisão de não implantar qualquer programa, ou seja, em 98,7\% desses hospitais, a decisão referente a iniciativas de qualidade ainda não se tinha destacado, trazendo à baila a questão do processo decisório em organizações hospitalares (Medeiros, 1999; Malik, 1983). No caso em que foi tomada a decisão da não-implantação, os motivos alegados foram a falta de recursos, o alto custo da implantação, a demora na obtenção de resultados e a não-necessidade de se dispor desse tipo de atividade. Nos outros 74, ou a questão realmente não se tinha ressaltado, ou era mais um caso em que assuntos eram expostos, e a decisão a seu respeito postergada até que alguma força externa ou interna - solucionasse o impasse.

\section{NO SETOR SAÚDE, CUJOS}

\section{CUSTOS SÃO ALTOS E}

\section{AS RECEITAS SÃO SEMPRE \\ CONSIDERADAS INSUFICIENTES, \\ O DESCONHECIMENTO A}

\section{RESPEITO DE CUSTOS DE}

\section{DIFERENTES PROGRAMAS}

\section{É PREOCUPANTE.}

Entre esses 75 hospitais, 54 dos respondentes manifestaram a intenção de implantar algum programa. No entanto, destes 54, quando perguntados quanto pretendiam/imaginavam investir, 27 não sabiam quanto investiriam em treinamento, 24 não sabiam quanto investiriam em área física e em consultoria e 20 não sabiam quanto investiriam em informatização. Cinco respondentes pretendiam não investir nada em treinamento e informatização, seis em transformação física e oito em consultoria. Treze dos respondentes acreditavam ter de investir mais de $\mathrm{R} \$ 100.000,00$ em transformação física. Em treinamento, 13 acreditavam ter de investir menos de $\mathrm{R} \$ 10.000,00$; em informatização, a maior freqüência foi 10 , concentrada também em menos de $\mathrm{R} \$ 10.000,00$ e, em consultoria, até $\mathrm{R} \$ 30.000,00$ foi a resposta de 17 . Mais uma vez, a preocupação com custos não parece ter chegado de fato ao ambiente hospitalar.

\section{CONCLUSÕES}

Os resultados obtidos apontam para o conhecimento e interesse abaixo do esperado, na era da globalização, a respeito de um dos modismos do final dos anos 80 e de todos os anos 90 .

Qualidade deveria ser uma reivindicação geral na área da assistência médica. Seria assunto de interesse dos cidadãos, que, embora ainda raramente, começam a reclamar perante os resultados insatisfatórios ou os custos abusivos. Já está no discurso dos financiadores, que re- 
clamam dos custos da assistência e se queixam de serem confrontados com "caixas pretas", sem conseguir diferenciar o que mereceria ser remunerado de maneira diferenciada daquilo que é feito até mesmo sem necessidade. Os profissionais do setor aprendem em seu processo de formação a defender "qualidade a qualquer preço". O poder público tem começado a se movimentar a fim de ouvir mais a população e de estimular o desenvolvimento de programas de qualidade nos seus serviços próprios, embora os investimentos para isto ainda tenham de ser buscados mediante projetos especiais. As principais conclusões da pesquisa são expostas a seguir.

Talvez "programas ou iniciativas de qualidade" não possam ser considerados, no Brasil, na área de saúde, como vantagem competitiva ou necessidade para entrar no mercado. Muitos afirmam que não existe mercado em saúde, mas, para os que acreditam nele, pode ser que ou o mercado de saúde ainda não seja/esteja competitivo ou que serviços de saúde tenham outros mecanismos para se mostrar competentes, mesmo contrariando alguns achados da literatura (Harrington, 1997). Saúde como um bem de mérito ou a precedência da eficácia sobre a eficiência é outra leitura possível para a realidade atualmente percebida (Julien, 1998).

Pode não haver desejo real por mudança nas organizações hospitalares. Ela costuma ser vista como necessária em face de problemas ou induzida por causas claramente perceptíveis. Se isto não ocorre, há lugar para a falta de vontade de pensar em mudar tanto a organização quanto sua cultura (Malik, 1992; Whitney e Pavett, 1998; Laszlo, 1998; Cameron e Sine, 1999). Também se torna plausível o medo dos efeitos de atividades voltadas à qualidade nas organizações (Moran e Brightman, 1998), inclusive pensando na atuação de avaliadores externos (Romano, Rainwater e Antonius, 1999).

Dados encontrados na bibliografia permitem inferir que ocorre resistência dos administradores à gestão da/pela qualidade (Feinberg, 1998). Há críticas constantes no Brasil à atuação não "profissional" dos administradores de setor, tanto na área privada quanto na pública. Por outro lado, em hospitais, há quem perceba importante resistência dos médicos a mudanças em geral e a programas de qualidade em particular (Pena, 2000; Terra, 2000).

$\mathrm{O}$ "desconhecimento profundo" (e assumido) dos custos da chamada gestão da qualidade, constatado nesta pesquisa, não difere do desconhecimento quanto aos custos nas atividades de saúde de maneira geral (Laszlo, 1997; Machowski e Dale, 1998).

Faltam argumentos para afirmar que a "qualidade" melhorará o desempenho das organizações de saúde ou que ajudará a alcançar maior envolvimento dos trabalhadores ou mais satisfação da população usuária (Hardie, 1998; Gustafsson e Johnson, 1997; Huff, Fornell e Anderson, 1996; Guimaraes, 1996; Jayram, Handfield e Ghosh, 1997; Wood Jr. e Urdan, 1994; Tolovi Jr., 1994).
Não há sequer evidências de que os custos baixarão, pois a maior parte dos fatores que incide sobre eles é externa aos hospitais. No Brasil, é recente a percepção das mudanças na atuação das operadoras/financiadoras em suas relações com os hospitais, embora todos os envolvidos no processo se queixem de problemas econômicofinanceiros (Jarlier e Charvet-Protat, 2000).

A implantação de programas/iniciativas de qualidade pode não ser considerada relevante para os respondentes, idealmente gerentes hospitalares. Talvez isso ocorra pela inexistência de pressão social suficiente para obrigar os hospitais a se preocuparem com os indicadores que apresentam, sejam eles financeiros, de resultados clínicos ou até de satisfação de seus usuários (Cecilio, 1999). Por outro lado, o discurso de escassez de recursos entre os dirigentes hospitalares é convincente o suficiente - pelo menos internamente ao setor - para justificar a falta de investimentos em operações consideradas por eles como "não essenciais". Por mais que se preconize que as atividades de qualidade devam fazer parte regular das tarefas de todos os trabalhadores, observa-se, na área da saúde, que ou se designam responsáveis por esse tipo de conteúdo ou não se conseguem implantar "programas/iniciativas" (Berwick, 1994; Terra, 2000).

Entre as experiências conhecidas pela maioria dos respondentes, estão as desenvolvidas por hospitais considerados "ricos" (Terra, 2000), cujos modelos podem ser vistos como inatingíveis para a maioria dos hospitais que ainda não se dispuseram a iniciar esse processo. Finalmente, o baixo nível de respostas pode se dever à metodologia utilizada. Os termos "programas" e "atividades" de qualidade podem ter sido percebidos como definições formais.

Talvez haja "esforços" de qualidade empreendidos que foram desconsiderados por não estarem formalizados. A experiência permite relatar, em serviços de saúde, a ocorrência de diversas iniciativas sem conhecimento da direção superior (por vezes até à sua revelia). O instrumento foi aplicado a um respondente quase com certeza ligado à direção superior. Se pode ocorrer de a direção superior, seus assessores e até conselheiros desconhecerem o cotidiano de unidades de prestação direta de serviços, que dizer da implantação de programas em unidades específicas?

\section{COMENTÁRIOS FINAIS}

Este estudo conseguiu cobrir uma amostra dos hospitais do estado de São Paulo. No entanto, o fato de apenas $61 \%$ dos questionários enviados terem sido respondidos demonstra um viés. A uma consulta telefônica feita aos hospitais de pequeno porte (sub-representados entre os respondentes), obteve-se a informação de que, entre eles, a porcentagem de programas/iniciativas era menor que 
na amostra como um todo. Assim, uma próxima investigação nessa linha deveria considerar essa limitação. A validade desta pesquisa é para o estado de São Paulo e para as condições de compra/venda de serviços vigentes nos anos 1999-2001. Nova pesquisa pode ser realizada num curto período, para verificar se regras comerciais mais claras e maiores exigências do setor público podem mudar essa realidade. Outra linha de trabalho a ser desenvolvida pode ser descobrir quais (de fato) são os indicadores usados nos hospitais. $\bigcirc$

\section{REFERÊNCIAS BIBLIOGRÁFICAS}

AZEVEDO, C. da S. Gestão hospitalar: a visão dos diretores de hospitais públicos do município do Rio de Janeiro. Revista de Administração Pública, Rio de Janeiro, v. 29, n. 3, p. 33-58, 1995

AZEVEDO, A. C. de, KORYCAN, T. L. Transformar las organizaciones de salud por la calidad. Santiago : Parnassah, 1999.

BERWICK, D. M. et al. Melhorando a qualidade dos serviços médicos, hospitalares e da saúde. São Paulo: Makron Books do Brasil, 1994

BITTAR, O. J. N. Qualidade e produtividade em hospitais. São Paulo : Sarvier, 1997.

CAMERON, K., SINE, W. A framework for organizational quality culture. Quality Management Journal 99, v. 6, n. 4, p. 7-25, 1999.

CARAPINHEIRO, G. Saberes e poderes no hospital: uma sociologia dos serviços hospitalares. Porto : Edições Afrontamento, 1993

CECILIO, L. C. de O. O Estado como prestador direto da assistência hospitalar. Revista de Administração Pública, v. 33, n. 2, p. 23-37, 1999.

CIS. Cadastro Geral dos Hospitais de São Paulo. São Paulo, Secretaria de Estado da Saúde, 1998.

DEMING, W. E. Qualidade: a revolução na administração. São Paulo : Marques-Saraiva, 1990.

FEINBERG, S. Why managers oppose TQM. The TQM Magazine, v. 10, n. 1, p. 16-19, 1998.

FERREIRA, J. H. G. Alianças estratégicas em hospitais privados. Tese (Doutorado) - Faculdade de Saúde Pública, Universidade de São Paulo, 2000.

GONÇALVES, E. L. Estrutura organizacional do hospital moderno. RAE - Revista de Administração de Empresas, v. 38, n. 1, p. 80-90, jan./mar.1998.

GORMAN, R. F., KREHBIEL, T. C. Quality management and sustainability. Quality Management Journal 97, v. 4, n. 4, p. 8-15, 1997.

GUIMARAES, T. TQM's impact on employees attitudes. The TQM Magazine, v. 8, n. 1, p. 20-25, 1996.

GUSTAFSSON, A., JOHNSON, M. D. Bridging the quality-satisfaction gap. Quality Management Journal, v. 4, n. 3, p. 27-43, 1997.
HARDIE, N. The effects of quality on business performance. Quality Management Journal 98, v. 5, n. 3, p. $65-83,1998$

HARRINGTON, H. J. The fallacy of universal best practices. The TQM Magazine, v. 9, n. 1, p. 61-75, 1997.

HUFF, L., FORNELL, C., ANDERSON, E. Quality and productivity: contradictory and complementary. Quality Management Journal 96, v. 4, n. 1, p. 22-39, 1996.

JARLIER, A., CHARVET-PROTAT, S. Can improving quality decrease hospital costs? International Journal for Quality in Health Care, v. 12, n. 2, p. 125-133, 2000.

JAYARAM, J., HANDFIELD, R., GHOSH, S. The application of quality tools in achieving quality attributes and strategies. Quality Management Journal 97, v. 5, n. 1, p. 75-100, 1997.

JULIEN, F. J. Tratado da eficácia. São Paulo : Editora 34, 1998.

LASZLO, G. P. The role of quality costs in TQM. The TQM Magazine, v. 9, n. 6, p. 410-413, 1997.

LASZLO, G. P. Implementing a quality management program - three Cs of success: commitment, culture, cost. The TQM Magazine, v. 10, n. 4, p. 281-287, 1998.

MACHOWSKI, F., DALE, B. G. Quality costing: an examination of knowledge, attitudes and perceptions. Quality Management Journal, v. 5, n. 3, p. 84-95, 1998.

MALIK, A. M. Processo decisório em instituições de saúde: estudo de algumas variáveis em hospitais complexos no município de São Paulo. Dissertação (Mestrado) - Escola de Administração de Empresas de São Paulo, Fundação Getulio Vargas, 1983.

MALIK, A. M. Desenvolvimento de recursos humanos, gerência de qualidade e cultura das organizações de saúde. RAE - Revista de Administração de Empresas, v. 32, n. 3, p. 32-41, jul./ago. 1992.

MALIK, A. M., SCHIESARI, L. M. C. Qualidade na gestão local de serviços e ações de saúde. São Paulo : Peirópolis, 1998.

MALIK, A. M., TELES, J. P. Qualidade em saúde. São Paulo : FGV-EAESP, 2000. Projeto de pesquisa financiado pelo NPP.

MEDEIROS, R. A. Processo decisório em hospitais: estudo descritivo em hospitais de grande porte no município de São Paulo. Dissertação (Mestrado) - Faculdade de Administração, Pontifícia Universidade Católica de São Paulo, 1999.
MORAN, J. W., BRIGHTMAN, B. K. Effective management of healthcare change. The TQM magazine, v. 10, n. 1, p 27-29, 1998.

PENA, F. P. M. Implantação do modelo ISO 9002 na área da saúde: a visão do gestor da qualidade em quatro unidades de um hospital no Município de São Paulo. Dissertação (Mestrado) - Escola de Administração de Empresas de São Paulo, Fundação Getulio Vargas, 1999.

PICCHIAI, D. Mudança em instituições hospitalares: análise do processo e estudo da ação do administrador hospitalar. São Paulo : FGV-EAESP, 1998. NPP, Relatório n. 4.

ROMANO, P. S., RAINWATER, J. A., ANTONIUS, D. Grading the graders: how hospitals in California and New York perceive and interpret their report cards. Medical Care, v. 37, n. 3, p. 295-306, 1999.

SCHIESARI, L. M. C. Cenário da Acreditação Hospitalar no Brasil: evolução histórica e referências externas. Dissertação (Mestrado) - Faculdade de Saúde Pública, Universidade de São Paulo, 1999.

SHORTELL, S., KALUZNY, A. Health care manangement: a text in organizational theory. New York : John Wiley \& Sons, 1998.

SILVA, U. S. Indicadores de qualidade e produtividade. Dissertação (Mestrado) - Escola de Administração de Empresas de São Paulo, Fundação Getulio Vargas, 1998.

TERRA, V. Mudança organizacional e implantação de um programa de qualidade em um hospital do município de São Paulo. Dissertação (Mestrado) Escola de Administração de Empresas de São Paulo, Fundação Getulio Vargas, 2000.

TOLOVI JR, J. Por que os programas de qualidade falham? RAE - Revista de Administração de Empresas, v. 34, n. 6, p. 6-11, nov./dez. 1994.

WHITNEY, G., PAVETT, C. Total quality management as an organizational change: predictors of successful implementation. Quality Management Journal, v. 5, n. 4, p. 9-22, 1998.

WOOD JR., T., URDAN, F. T. Gerenciamento da qualidade total: uma visão crítica. RAE - Revista de Administração de Empresas, v. 34, n. 6, p. 45-59, nov./dez. 1994.

\section{NOTAS}

Este artigo baseia-se em pesquisa financiada pelo NPP - Núcleo de Pesquisas e Publicações da FGV-EAESP intitulada "Programas de qualidade em hospitais".

Agradecemos, pelo apoio metodológico, aos professores Maria Christina S. Souza Campos e Wilton de Oliveira
Bussab. À primeira, pela valiosa assessoria na elaboração da abordagem metodológica, do questionário de pesquisa e pela leitura atenta do primeiro esboço do projeto, bem como da primeira versão do relatório. Ao segundo, pela orientação na determinação da amostra, nos métodos estatísticos que foram empregados e pelo auxílio na análise dos resultados (principalmente na compreensão de sua validade). Pela cuidadosa leitura e pelas sugestões recebidas, agradecemos a Laura Maria Cesar Schiesari, Paula Carnevale Vianna, José Maurício de Oliveira, Everton Soeiro e H. Maria D. Novaes. Cada uma dessas leituras nos permitiu um olhar diferente sobre nossos achados. 